\title{
Namenregister zu Band 90
}

Abramowicz 57, 323 Agnello 202, 205 Akita 314 Alabaster 282 Alajmo 40 Allmaras 114 Altland 113 Alvaro 284 Anastasi 312 An din a 50 Anelli 320 Applemann 44 Argentino 55 Arjona 40 Arkin 212 Arnold 38 Arruga 383 Ascher 283 Aubineau 296 Aujalen 381 Aust 328 Baba 54 Bach 218 Baenzinger 49 Baer 311 v. Bahr 68 Baillart 210 Bakker 40 Balado 355, 358 Balcet 320 Bannwarth 367 Barber 312 Bard 291 Barkan 319 Barrata 39, 53, 323 del Barrio 210 Bartels 66, 115, 119,

216, 227, 228, 374 Bartolotta 343 Bau-Prussak 365 Baurmann 50, 51, 82 Becker 40 Beckh 199 Behr 350 Belgeri 316 Bellavia 39 Bender 311 Benoit 328 Bérard 316 Berens 212, 281, 283 Berg, F. 240 Bergler 6 Bernhard 222 Bertel 57 Bielschowsky 280, 284, 286

Bienvenue 363 Bietti 36 Biffis 45 Le Bigot 363 Biozzi 42 Biró 40, 203 Bistis 13 Blaickner 72 Blaskovics 209 Boardmann 42 Bock 100 Böck 204 Bokermann 115 van Bogaert 365 Bologowa 341 Borsellino 43 Bourno 37 Braun 37, 42, 78, 103 Brockmayer 216 Brody 358 Brons 117 Brunner 219, 220, 221 Brunton 52 Bryngelson 282 Bucklers 35, 63, 98.

103 Bufill 322 Bujadoux 322 Busacca 206, 337 Busch 367 Buschke 36

Cacioppo 45 Campos 35 Caprano 35 Cardello 324 Castresana 205 Cattaneo 339 Cavaniglia 205 Chavasse 282 Chini 287 Churgina 197 Chworow 56 Ciaccio 34 Clausen 61, 71 Coenen 364

Colin 318 Colley 54 Collins 357 Colombies 381 Colrat 286 Comberg 70, 74, 102 Cuénod 336, 343 Cushing 366 Custodis 90, 228, 369

Daniel 42 David 367

Davson 47 Dejean 318 Delord 318 Demeler 115 Demole 36 Denig 44 Dercac 319 Derer 45

Desplas 374 Devorah 327 Diaz-Dominguez

210 Dimissianos 331 Dodge 38

ten Doesschate 125 Dollfus 239 Dopheide 193 Ducourtioux 316 Dudinow 196, 323 Duke-Elder 47 Dunphy 203 Dusseldorp 54 Dyes 58

Eagleton 52 El Bakly 317 Elliot 210 El Tobgy 317 Engelking 69 Engerth 361 Erggelet 81 Esaka 37

EspildoraLuque37i Esser 317 Euler 37 Evans 196, 359

Fahmy 323 Fanchamps 203 Fantus 204 Fazakas 332 di Fede 45 Ferraris 57 Fésus 332 Le Fever 367 Fine 285 Fink 282 Fischer, F. P. 38,

214, 248, 355, 357 Fiszmann 322 Fleischer 59, 69, 374 Focosi 50 Fodor 49 Fontana 206, 339

Fox 359

Fradkin 36, 211 Francois 329 Frank 319

$390 \mathrm{~J}$

Franke, E. 358 Frey 222 Freyler 226 Friedenwald 48 Fromm 216 Frost 353 Fuchs, A. 291

Gabriélidès 43 Garcia-Miranda 282 Gareiso 363 Gasser 49

Gasteiger 59, 85, 106 Gastew 232 Genet 351 Gerhartz 363 Germain 326 German 359 Ghio 54 Gifford 283 Gillies 319 Globus 368 Gödl 202 Goedbloed 48 Goldberg 222, 363 Goldfeder35, 45,319 , 
325 Goldmann 36, 42 Golowin 54 Gomez-Marquez355 Gordon 368 Gotoh 53 Gouin 363

Grabscheid 220 Gradle 208 Graff 81 Grahe 221 Grandi 282 Granström 203 Grimm 281 v.

Grolman 41 Grönlund 288 Grosz 207 Grüter 88, 108, 338 Grz§dzielski 43 Gudzent 123 Guleke

238 Gummerus 196 Gutsch 96 Gutzeit 120 György 37

Haas 57 Haene 369 Haessler 281 Hagedoorn 312, 313,

357 Halász 45 Haldimann 146 Hamburger, C. 206 Hamburger, F. A.

225 Hamilton 382 Hansing 204

amenregister zu Band 90

Harms 81, 101, 102,

279 Harris 37 Harrison 337 Hartinger 100 Hartmann 367 Hartshone 44 Hata 211 Heinonen 41, 330 Heinsius 266 v. Herrenschwand

33i Hertel 70 van Heuven 325 Le Heux 216 Hicks 282, 320 Higuchi 329 Hildreth 318 Hitta 45

van der Hoeve 75 vom Hofe 93, 105 Hoff 360, 361 Hoffmann, W. 55 Hollander 311 Holloway

366 Holtz 93 Horay 283 Horváth 284 Hosford 282, 320 Hossmann 312 Howard 207 Huber 41

Hubert 284 Huizinga 218

Itayama 196 Isoh 288 Iwanow 313 Iwasawa 314 Iwaszkiewicz 57

Jaensch 35, 49, 231 Jäger 35 James 320 Jameson 283 Jancke 109, 119, 279 Jansen 37 Jeandelize 358 Jean-Gallois 207 Jenkins 53 Jess 64, 97 Johansson 31, 301 Johns 327 Joiris 203 Joseph 209 Josephson 42, 206 Juba 359 Judkewitsch 39 Jufa 212 Julianelle 337 Jung 58 Jusefowa 57 j Just Tiscornia 40

Kafka 113 Kahoun 284 Kalt 54 Kaplan 42 Kapuscinski 44 Karalitz 351 Karsch 157, 316

Kawasaki 53 Kesselyák 34 Kestenbaum 214, 220, 294 Keyes 58 Khorazo 327 Kiewe 295, 312 Kiskadden 319 Klar 374 Klein 287 Kleist 361 de Kleyn 216 Knapp 44

Kogan-Abeshus 342 Kojima 49 Kokott 50 Kolen 317 Kopp 317 Köppl 226 Kornblum 57 Kotlj arewskaj a 210 Kourilsky 374 Kowalew 371 Koyanagi 354 Kraupa 32, 128, 192,

201, 273, 276, 310 Krause, A. C. 35, 49 Kreibigl29, 225, 290,

335 Kreiker 325 Kreimer 38 Krepuska 221 Kreyenberg 204 Krückmann 91, 229,

281, 311 Krylow 205 Kugelberg 322 Kukán 166, 202, 315 Kulkow 371 Kumamoto 325 Küper

40 Kurz 40, 200 Kyrieleis 58, 100,

101,350

Labbé 52 Lacat 209 Lagrange, A. 121 Lagrange, H. 44., 121 Laignier 210 Lapierre 314 Laszlo

205 Lauber 83, 84, 356 Lavagna 39 Lavial 324 Layton 53

Namenregister zu Band 90.

391

Lebras 284 Lehotzky 39 Leidler 220 Leinfelder 52 Lester 311 Levy, M. 284 Lewine 211

Liebermann 44 Lindberg 370 Lindner 50, 60, 101,

223, 224, 227, 294 Lobeck 99 Lo Cascio 43 Loepp 55, 57 Löhlein 60, 67 Löwenstein 337

Ludwig 51, 314 Lugli 43 Lukiesh 281 Lusza 316

Macht 342 Mackiewicz 365 Magnusson 316 Malbran 355, 358 Malmberg 37 Manes 45, 197

Mann 34, 56 Marburg 380 Marchesani 54, 71,

78, 35i, 354- 373 Marcus 341 Mariotti 201 Markus 39 Marlow 281 Márquez 284 Marquézy 202

Martin 207 Marucci 314 Matsumoto 288 Mayer, E. G. 57 Mazzei 42 Mc Laurin 211 Mc Millan 359 Mecca 285 Meesmann 67, 94,101, 
108 Meisner 119, 228, 230 Meissner 90 Mendzerickij 53 Mengel 288 Mengert 338 Meredith 369 Mexina 34 Meyer, M. F. 53 Meyerhoff 328 Michail 54 Mikawa 41 Mikumi 361 Milner 283 Miloro 206 Mingelen 43 Minkin 18

Mirto 39 Mitchell 38 Mittermaier 220, 221 Mizkewitsch 338 Moeller 53, 297 Monjukowa 36, 41 Moorad 216 Morancé 57 Morax 331, 373 Moretti 207 Moren 44 Moss 281 Mossa 340 Motegi 339 Mourzinn 341 Mowrer 218 Mügge 70, 95 Müller, H. K. 35, 36, 73 Müller, L. 126 (P.) Müller-Stieber 232 Mulock Houwer 43, 201 Mura 39, 317, 339 Muskens 219 Mylius 76

IVakamara 337 Nataf 343 Natale 314 Neumann 334 Newton 320 Ngowyang 362 Nicolini 315 Niessl v. Mayendorf

241 Niimi 325 Nikolajewa 197 Nizetic 122, 30ó de No 219 Nordlöw 282 Nordmann 38, 198 Nordsted 321 Nowkirischky 314 Nylén 216 Nyquist 206

O’Brien 38, 52 O'Connor 211, 281,

286 Oehlecker 60 Oguchi 342 Ohm 118, 215, 216, 218, 280 Ohno 313 Olitsky 336 Oliver 52 Olloz 371 Orlow 44 Orth 312 Oshima 332 Otto 327 Ovio 383

Padovani 203 Paget 38 Pallestrini 220 Pancoast 57 Panico 280, 319 Papadato 370 Papolczy 315 Passow 205 Paton 45, 283 Paul 100 Pavia 35 Pedersen 370 Penard 360 Penfield 359 Pera 53 Perera 203 Pergola 315, 340 Peters 339 de Petri 51 Petroff 288 Philippow 209 Philips 336 Pickard 122 Pietra 53 Pillat 79 Pirot 326 Pitha 287 Pittenger 40 Plaats 316 Plummer 51 Pockley 45 Pokrowskij 209 Pol 281 Pollock 283 Ponthus 316 Poos 118, 365 Popow 44 Posthumus 330 Pötzl 360, 361 Prangen 283 Proctor 336 Protopopow 341 Puglisi-Duranti 50 Pyke 37

Quin t 124, 23 Quix 219

Rabinowitsch 207, 209 Ramach Ill Rand 369 Rauh 94 Raverdino 44 Ray 37 Rebattu 286 Redslob 48, 198 Reese 321 Reh 295 Reichling 86, 104,

107 Reimova 320 Reiners 231

392

Namenregister zu Band 90.

Reiser 89, 231 Reiss 38, 48 Rempel 324 Rice 339 Richards 336 Ridley 320 Rieger 80, 104, in Riehm 92, 364 Rintelen 196, 315 Riser 322 Rist 331 Roberts 371 Robertson 47 RochonDuvigneaud 35 Rodin 319 Roeling 53 Roelofs 217 Roggenkämper 116, 229 Rohrschneider 105 , 119, 196, 230, 231 Rollin 311, 325 Romanowa-Bochon Römmelt 1 Rönne 102 Rosengren 197, 322 Rössle 120 Rössler 293 v. Rötth 216 Rubert 343 Rubino 40, 41 Rubio 329 Rugg-Gunn 281 Rusco 199 Russo 40

Sáenz-Alonso 324

Safár 112

Sai 37

Salit 38

Sallmann no, 212, 224, 294 Salvati 199 Santha 287, 367 Santoro Vecini 353 Sargnon 57 Sato 34 Savin 202 Schaeffer 288, 371 Scherewskaj a 211 Schimanski 371 Schmelzer 95 Schmerer 34 Schmidt 103, 
228, 230 Schnyder 41, 42 Schornstein 321 Seefelder 41 Seidel 85, 106, 109 Seiferth 219

Selinger 45

Sellei 37 Sergent 374 Shoji 45 Shutes 42 Siegert 311 Silagni 287 Silverstone 368 Sjögren 40, 73 Skydsgaard 204 Smaltino 37 Sobánski 200, 356 Sokolanski 371 Sørensen 367 Souchkowa 341 Spektor 52, 56 Stadler 237 Stauder 373 Steegmann 371 Steffen 53 Stein 205, 287 Stengel 220, 372 Stenvers 217 Stewens 117 Stiehler 48 Stiel 263 Stier 221 Stock 65, 69 Strachow 44

Strampelli 35 Sundqvist 240 (P.) Susuki 311 Syverton 336 Szász 199 v. Szily 42, 337 Szolnoky 53

Takao 41 Talbot 339 Tanobe 284, 288 Taubenhaus 207 Tavennec 202 Taylor 279 Terrien 239, 354, 370 Terry 314 Terson 283 Tertsch 50 Thiebaut 374 Thompson 327 Thygeson 336, 338

Tichomirow 321 Tillé 370 Tolosa 285 Tönnis 369 Tovbin 208 Traquair 197 Tristaino 342

Troncoso 198 Trovatin 198 Trubin 340 Tschernikowa 57 Tsuchiya 49

Tumarkin 321 Tyler 336

Unterberger 221 Urban 361 Utkina 211

de Vecchi 39

Veil 239

Velhagen jung7,109,

355. 379 Vierling 125 Villani 35 Vito 313

Vittadini 284, 322 Vogel 351 Vogelsang 35, 105 yogt 35, 49, 234, 238

Waals 217 Waardenburg 61 van Wagenen 369 Wagner, R. 53, 334 Wainer 341 Walker 329

Wartenberg 233, 381 Wasowski 57, 323 Weber, F. P. 40 Weekers 314 Wegner 91, 109 Weil

198, 373 Weinstein 37, 199 Wenderowic 371 Werdenberg 107 Werpoukkowsky232 Wescott 297

Wessely 313 Weve 125 Wheeler 283 Wieczorek 55 Wiedersheim 216 Wilder 51 Wilson 340

Wirth 221 Wissmann 71 Wokurek 273 Wolfe 44

Wolokonenko 339 Worms 352 Wostrý 54 Wünsch-Heitz 259 Würdemann 296

Y. Yamagata 53 Yamamoto 222 Yudkin 38

Zacharowa 39 Zeeman 201

358 Zeiss 299 Zenker 222 Zikulenko 341 Zwick 313 